Meta

Journal des traducteurs

Translators' Journal

\title{
La traduction, phénomène interculturel et psychorelationnel
}

\section{Jean-René Ladmiral}

Volume 55, numéro 4, décembre 2010

De la localisation à la délocalisation - le facteur local en traduction

From Localization to Delocalization - The Local Factor in Translation

URI : https://id.erudit.org/iderudit/045682ar

DOI : https://doi.org/10.7202/045682ar

Aller au sommaire du numéro

Éditeur(s)

Les Presses de l'Université de Montréal

ISSN

0026-0452 (imprimé)

1492-1421 (numérique)

Découvrir la revue

Citer cet article

Ladmiral, J.-R. (2010). La traduction, phénomène interculturel et psychorelationnel. Meta, 55(4), 626-641. https://doi.org/10.7202/045682ar

\section{Résumé de l'article}

Les questions qui peuvent se poser à propos du concept de localisation amènent à problématiser l'idée de traduction. La communication mise en jeu dans le cadre d'une dynamique de groupes bilingues et binationaux (par exemple franco-allemands) prend la forme d'une " traduction " orale qui, au sein du groupe, devient l'enjeu des clivages linguistiques et interculturels, mais aussi de tout un vécu psychorelationnel. Sans doute le concept de médiation permet-il, par sa généralité et sa polysémie, d'appréhender les problèmes posés dans toute leur ampleur. Par en haut : le champ d'études inédit dont il est traité ici amène à ne pas éluder l'échéance d'un questionnement d'ordre épistémologique. Par en bas : plusieurs scénarios de communication interculturels seront évoqués. 


\title{
PARTIE I: PENSER LE LOCAL
}

\section{La traduction, phénomène interculturel et psychorelationnel}

\author{
JEAN-RENÉ LADMIRAL \\ ISIT, Paris, France \\ Université Paris X - Nanterre, Paris, France
}

\begin{abstract}
RÉSUMÉ
Les questions qui peuvent se poser à propos du concept de localisation amènent à problématiser l'idée de traduction. La communication mise en jeu dans le cadre d'une dynamique de groupes bilingues et binationaux (par exemple franco-allemands) prend la forme d'une «traduction» orale qui, au sein du groupe, devient l'enjeu des clivages linguistiques et interculturels, mais aussi de tout un vécu psychorelationnel. Sans doute le concept de médiation permet-il, par sa généralité et sa polysémie, d'appréhender les problèmes posés dans toute leur ampleur. Par en haut: le champ d'études inédit dont il est traité ici amène à ne pas éluder l'échéance d'un questionnement d'ordre épistémologique. Par en bas: plusieurs scénarios de communication interculturels seront évoqués.
\end{abstract}

\begin{abstract}
The issue which may arise about the concept of localization leads us to question the notion of translation. The communication at stake within the dynamics of bilingual and dual-nationality groups (e.g., Franco-German groups) becomes a kind of oral translation which, within said group, will be based on linguistic and intercultural schisms, but also of a whole psycho-relational experience. The notion of mediation makes it possible through its all-encompassing nature and its polysemy to approach this particular field of study in its entirety. On a higher level, the unexplored field of study examined here hitherto leads to an evasion of the ultimate epistemological reflection. On a lower level, several intercultural communication scenarios will be presented.
\end{abstract}

\section{MOTS CLÉS/KEYWORDS}

bilinguisme, communication interculturelle, épistémologie, médiation, scénarios de communication

bilingualism, intercultural communication, epistemology, mediation, communication scenarios

à Ewald Brass

\section{Approche conceptuelle}

Contrairement à ce qu'on serait porté à penser spontanément, le concept de traduction fait problème; et je dirai que le terme de localisation en est l'un des nombreux indices. S’agissant de la localisation, qui est au centre de la présente livraison de Meta, le mot lui-même appelle quelques précisions.

On a là une illustration de l'alternative que j'ai thématisée en traductologie sous le nom du «théorème de dichotomie», selon que l'item linguistique à traduire devra 
faire l'objet d'une terminologisation ou d'une idiomatisation (Ladmiral 1997). Ainsi le concept de localisation fait-il l'objet d'un clivage sémantique distinguant de son sens général et traditionnel un sens «technique» (ou, en l'occurrence, plusieurs), comme c'est le cas de nombreux vocables dans la langue.

$\mathrm{Au}$ sens technique, qui est aussi un sens restreint, c'est une unité terminologique (terminus technicus) qui représente une nouveauté et qu'on pourra qualifier de néologisme sémantique puisqu'il s'agit d'un signifiant ancien affecté d'un signifié nouveau. Pour simplifier, je dirai qu'il s'agit essentiellement de la traduction des logiciels informatiques. La question se pose (ou se posait) de savoir si ce néologisme s'imposait. On peut penser que non. Il y a quelques années, quand le terme a commencé à être attesté, certains d'entre nous étaient très réticents, comme Daniel Gouadec ou moi-même. Ne pouvait-on pas en rester au terme de traduction? Enrichi de ces données nouvelles, comme c'est au demeurant le fonctionnement normal du lexique de tout langage. Quoi qu'il en soit, il est clair qu'en matière de langue, c'est l'usage qui fait autorité en dernier ressort: dès lors qu'un terme s'est imposé dans la pratique, il convient de s'en arranger, quand bien même on persisterait à considérer qu'il n'est pas «bien formé»; et la terminologie ne fait guère qu'assez rarement exception à cette règle.

Afin de justifier l'emploi de cette innovation terminologique, on fera valoir que ladite localisation implique «en plus» divers aménagements, qu'il s'agisse de compléments de nature ergonomique, de la prise en compte des exigences du marché, de contraintes d'ordre technique, etc. Mais tous les traducteurs et tous les traductologues sérieux n'ont-ils pas toujours su que la traduction ne se limite en aucun cas au transfert interlinguistique, consistant à mettre «un mot pour un autre»? De même, on pourra souligner la différence importante qu'il y a entre les textes, qui sont rédigés dans des «langues naturelles», et les codes, qui sont des langages formels. Mais fautil pour autant tracer une limite tranchée entre la traduction, traitant des premiers, et la localisation, opérant sur les seconds? Alors que, dans la pratique, il y a des chevauchements constants. En tout cas, quoi qu'il en soit au bout du compte du terme retenu, il y a là un vrai sujet, une thématique nouvelle et spécifique, dont il était légitime qu'il fût traité ici.

Pour être complet, à côté du sens proprement technique qu'a pris le terme de localisation dans le domaine informatique, il faudrait évoquer le sens «technique», très spécialisé, qui est le sien en linguistique. Dans la logique de la sémantique cognitive, mais aussi de la théorie des opérations prédicatives et énonciatives (Culioli 1990-1999), le concept de localisation revêt en linguistique une signification abstraite qui renvoie à l'isotopie métalinguistique de l'espace (mais aussi du temps) comme champ notionnel et mobilise dans leur plus grande extension les concepts de repère et de repérage. Cela permet d'analyser en profondeur les verbes de mouvement, les causatifs, le fonctionnement des prépositions, etc. On trouvera là tout un trésor de réflexions et d'analyses très fines et sophistiquées portant sur le langage et les langues naturelles. Mais cela va dans la direction de ce que j'appelle une sémantique scientifique, dont le point d'application pourra sembler "microlinguistique», alors que c'est à l'approche plus empirique d'une sémantique phénoménologique globalisante de la pratique qu'en appelle la traductologie (Ladmiral 2006). C'est ce qui nous conduit à faire ici l'impasse sur cette problématique importante, qui devra être reprise sur nouveaux frais. 
Il reste que le concept de localisation conserve parallèlement son acception traditionnelle, avec une aire sémantique beaucoup plus étendue, désignant les diverses modalités de situation dans l'espace et, plus concrètement, les questions de territoire. Cela concerne aussi bien les réalités les plus concrètes et matérielles que des prolongements théoriques, voire spéculatifs, qui peuvent aller jusqu'à rejoindre la philosophie elle-même: qu'on pense à la «spatialisation» dont Bergson a fait la critique ou au concept de déterritorialisation (ainsi que de territorialisation) qu'a thématisé Deleuze $^{1}$, sans parler de l'isotopie métaphorique de l'«habiter» chez Heidegger qui prend chez lui la dimension ontologique d'un philosophème (Wohnen), etc. Plus récemment, le concept de localisation devrait se charger d'une signification renouvelée relevant de la philosophie politique, dans le cadre d'une critique du mondialisme débouchant sur la nécessaire revalorisation du «local», tant pour des raisons géopolitiques et ethnoculturelles, voire nationales, que pour des raisons économiques et last but not least écologiques. À titre personnel, je serais enclin à engager ma réflexion dans ces diverses directions, rejoignant en cela certaines des préoccupations de mes travaux philosophiques; mais il est clair que ce n'est pas vraiment le sujet. Toujours est-il que, pour ce qui nous concerne ici, il y aura lieu de prendre en compte deux significations bien différentes de ce concept de localisation. On aura donc, d'une part, la nouveauté du sens restreint et proprement technique qui regarde vers l'informatique et qu'on pourra dire professionnel (terminologisation); et puis il subsiste, d'autre part, une signification très large et plurivoque, qu'on pourrait dire culturelle et que j'ai envie d'appeler «linguistique» dans la mesure où c'est l'acception naturelle et non marquée que revêt le mot dans les différents contextes du langage courant ou même spécialisé (idiomatisation). Plus précisément, je préférerai parler plutôt de deux «pôles sémantiques» pour autant que l'articulation de ces deux sens constitue une polarité, c'est-à-dire un clivage très net, mais qui n'exclut pas totalement la transition d'un continuum intermédiaire. Tel est bien l'esprit des contributions rassemblées ici, qui ne se limitent pas à traiter des logiciels informatiques, relevant du seul domaine de la traductique. Le propos qui a été adopté est proprement traductologique et tend à prendre en compte des approches très variées dans l'espace ouvert par l'ambivalence de l'idée de localisation.

Par contrecoup, cette question de mot à propos de la localisation nous conduit à réexaminer le concept de traduction lui-même - qui, en dépit de tous les renouvellements que nous impose la modernité, reste quand même le «cœur de cible» de Meta. C'est ainsi qu'en l'occurrence j'y ai entrepris de thématiser la polysémie de ce «concept pluriel» (Ladmiral 1995), sans toutefois proposer en l'espèce une véritable typologie de la traduction (Reiss 2009). Inutile d'insister (ni d'épiloguer) sur la distinction binaire que l'on connaît trop, à savoir:

- la traduction professionnelle ou spécialisée (1) (Lavault 2007), que certains auteurs comme Jean Delisle ou Nicolas Froeliger appellent aussi traduction "pragmatique» (Delisle 1980: 20-21, Froeliger 2009: 201), alors que cette dernière appellation fait à mes yeux figure de germanisme;

- la traduction littéraire, ou plutôt la "traduction des cuvres» (2) dirai-je en reprenant la formule d'Antoine Berman, mais en lui donnant un sens élargi pour y intégrer aussi la traduction philosophique et la traduction des Textes sacrés.

Point n'est besoin non plus de revenir sur ce «degré réduit» de la traduction que constituent les diverses formes que peut prendre la traduction dans l'institution 
pédagogique (3) (voir notamment Ladmiral 1994: 23-83). Je mentionnerai au passage la dite traduction orale (4), traditionnellement appelée interprétariat et récemment rebaptisée interprétation (pour différentes raisons, en grande partie "promotionnelles»), dont je préfère parler en disant le «travail de l'interprète» pour éviter les querelles de mots.

Pour compléter ce bref récapitulatif et entrer plus spécifiquement dans le vif du sujet, j'évoquerai encore deux figures particulières de la traduction. Dans la perspective du sens large (idiomatisant) que revêt au départ le terme de localisation, je m’attacherai dans la suite de la présente étude à traiter d'une modalité tout à fait particulière de la "traduction" comme phénomène interculturel et psychorelationnel (5). Mais auparavant, j'entends revenir brièvement sur le sens restreint (terminologisant) de localisation et sur certaines réflexions qu'appellent ses implications. Ainsi qu'il a été indiqué, le terme lui-même constitue (6) un synonyme a contrario (ou in absentia) du concept de traduction - spécifié, il est vrai, au domaine d'application très particulier de l'informatique -, mais il reste que c'en est le signifié sans le signifiant. Dès lors se pose la question de savoir pourquoi il a été procédé à ce qu'on peut appeler en l'occurrence un «détournement onomasiologique». Sans doute y a-t-il à cela plusieurs raisons.

À première vue, on peut y voir un exemple de plus de cette "valse des étiquettes", qui veut qu'on cherche toujours des appellations inédites pour vanter la nouveauté d'un produit qu'on veut lancer sur le marché. Cette façon de faire qui nous vient de la publicité commerciale est tout naturellement pratiquée dans le secteur des productions technologiques plus ou moins récentes; mais elle s'est aussi répandue assez largement dans le discours des sciences humaines (notamment dans les pays anglosaxons) et, à la marge, elle a commencé à «grignoter» certaines spécialités proprement scientifiques. Or, dans la pratique, la traduction (et en particulier celle de logiciels) est bien évidemment à la croisée de l'ensemble de ces différents domaines. D’une façon générale, on n'est que trop porté à faire du neuf avec du vieux. À quoi peuvent venir s'ajouter les tentations élitistes d'un certain ésotérisme terminologique, censé faire ressortir la compétence de ceux qui en ont la pratique, un peu comme le latin des médecins de Molière.

Mais l'essentiel n'est pas là. C'est l'idée même de traduction qui est en cause. Chez ceux qui n'en ont pas fait l'expérience, dans la réalité de la pratique, il y a une incroyable méconnaissance du problème. C'est ce que j'avais appelé l'oubli de la traduction, en donnant à l'«oubli» le sens fort qu'il peut prendre chez Freud et Heidegger (Ladmiral 2004). On pourra n'y voir a minima que l'effet d'une grande naïveté. D'aucuns, quand ils ont besoin d'une traduction, donnent l'original à « un étudiant» (en deuxième année d'anglais par exemple!) pour qu'il le traduise et ils s'étonneront ensuite de la médiocrité de la «traduction» qui leur sera fournie... Au reste, comme on sait, cette méconnaissance vient à point nommé pour justifier que les traducteurs sont sous-payés. Ce qui est moins vrai qu'ailleurs dans un pays comme le Canada, où il y a heureusement une logique institutionnelle de promotion de la traduction.

Cela dit, au-delà de cette (double) explication par défaut, j'ai la conviction qu'en cette affaire des motivations plus profondes sont à l'œuvre (par excès, pour ainsi dire). Je hasarderai le diagnostic qu'il existe chez beaucoup d'individus une ambivalence foncière à l'endroit des souvenirs qu'ils ont gardés de leur passé scolaire, comme j'ai 
eu la surprise de le constater chez tant de mes contemporains. Sans doute conviendrait-il d'aller jusqu'à l'idée, paradoxale et dérangeante, d'une ambivalence vis-à-vis de l'enfance elle-même. Mais cela débouche sur la double logique d'une psychologie des profondeurs et d'une philosophie des histoires de vie (de Descartes à Freud) qui, à l'évidence, excède les limites de mon propos ici (et qui fera l'objet d'une prochaine étude). Quoi qu'il en soit, il est indéniable qu'il y a fondamentalement un déni de la traduction - en dépit de tous les discours contraires qui peuvent être tenus, et qui font dès lors figure de ce qu'il y a lieu d'appeler justement des dénégations. Pour «les gens", le traducteur (et la traductrice), c'est l'homme invisible!

Je m'en tiendrai ici à un niveau élémentaire de cette problématique complexe. Pour ceux qui découvrent sur le tard et sans préparation spécifique ce qu'est réellement la traduction, il leur apparaît que ça n'a rien à voir avec les souvenirs flous et assez largement négatifs qu'il leur reste des thèmes/versions qu'ils ont dû ânonner en tant qu'élèves, dans des langues qu'ils ne maîtrisaient pas encore; et c'est dans le clair-obscur de cette expérience malheureuse qu'ils entendent le sens du mot «traduction ». Quand ils en sont venus plus tard à traduire un logiciel, par exemple, ils mesurent alors combien leur tâche est délicate et difficile, combien elle présuppose de connaissances multiples et exige de créativité, etc. Du coup, ce travail de qualité qu'ils ont conscience d'avoir accompli, ça ne peut pas être de la traduction! Cette activité mineure et subalterne qu'est une vulgaire traduction. Pour faire droit à la valeur ajoutée de leur travail, il faut donc un mot nouveau. En l'espèce, ce sera celui de «localisation». On pourra aussi parler de "médiation interlinguistique», de "management interculturel», de "transfert du sens", voire de "tradaptation", etc. Au demeurant, Cicéron lui-même affirmait déjà qu'il n'avait pas traduit les grands orateurs grecs comme un pur et simple traducteur (ut interpres), mais comme un écrivain (ut orator).

\section{La recherche}

\subsection{Le dispositif}

En deçà (pour ainsi dire) des modalités relativement formalisées de traduction (lato sensu) qui viennent d'être récapitulées rapidement, mon propos est d'aborder ici une forme particulière de gestion des clivages de langues que je n'ai fait que mentionner plus haut et où interfèrent massivement les surdéterminations interculturelles ainsi qu'une dynamique psychologique des relations entre individus.

Ces phénomènes ont pu être étudiés dans le cadre d'une recherche qui portait essentiellement sur la problématique de la dynamique des groupes bilingues dont j'ai eu l'occasion de publier des éléments de bilan intermédiaire en différents lieux, et notamment sous la forme d'une présentation générale parue en son temps dans Meta (Ladmiral 1982). Pour éviter de me répéter (et de lasser le lecteur éventuel), je ne rappellerai ici que les linéaments de la méthodologie mise en œuvre.

Il s'agissait d'un programme de recherche financé par l'Office franco-allemand pour la jeunesse (OFAJ) auquel il m’a été donné de participer comme chercheuranimateur et dont j'avais eu le privilège d'être l'instigateur au départ. Le dispositif général consistait à mettre en place un suivi de plusieurs stages résidentiels réunissant une vingtaine de jeunes Allemands et Français pendant une bonne semaine ou un 
peu plus, dans une perspective s'inspirant de la méthodologie de la dynamique des groupes restreints, augmentée d'un certain nombre de compléments d'ordre pratique et méthodologique qu'exigeaient la thématique de recherche adoptée et la spécificité des groupes en présence. La méthodologie de la dynamique de groupes était en partie infléchie par une re-contextualisation sociologique dans la perspective de l'Analyse institutionnelle (AI); et le type de groupes concernés étaient ce qu'il est convenu d'appeler des groupes de base ou groupes de thérapie (T-groups). Ce programme est maintenant clos: il a donné lieu à un rapport final édité par l'OFAJ et, par suite, à diverses publications (Ladmiral et Lipiansky 1989).

Dans ces groupes, il y avait bien évidemment des «bilingues», mais aussi très expressément des unilingues, des deux côtés (français et allemand), dans la mesure où il s'agissait de mettre en évidence comment les choses se passent pour que puisse s'établir la communication entre des gens qui ne parlent pas la même langue et qui participent d'horizons culturels différents. Pour quelqu'un de non averti, comme beaucoup de ceux auxquels j'ai pu parler incidemment de cette expérience, il «suffit» de prendre un interprète, et puis voilà! À quoi il y a lieu d'objecter trois ou quatre considérations. D’abord: un interprète, il faut le payer; et puis il en faut plusieurs pour couvrir un dispositif de cette ampleur. Il y avait donc là une impossibilité matérielle; car le financement du programme n'était pas illimité, d'autant qu'il prenait déjà en charge les frais de séjour de tous les participants et leur frais de voyage, à quoi venaient s'ajouter les honoraires des chercheurs-animateurs. Surtout, il convenait d'écarter a priori la "solution technique de l'interprète » parce qu'elle était en totale contradiction avec la logique de la recherche.

L'enjeu était en effet d'analyser par quels canaux et selon quelles modalités peuvent advenir des interactions verbales (et non verbales) entre les membres de tels groupes en dépit des obstacles linguistiques et des clivages interculturels, voire des oppositions nationales - ou, peut-être aussi, grâce à eux... Le choix de la méthode adoptée en faveur de la dynamique des groupes pour étudier ces deux variables s'insère très directement dans la problématique dont nous avons campé l'alternative dès le début de notre introduction au présent numéro quand nous avons inscrit la traduction à l'articulation du temps et de l'espace. Dans cet esprit, on peut observer que ladite dynamique problématise la dimension de la temporalité: le temps des groupes restreints dans un stage est une séquence de temps "hors temps», c'est une enclave microtemporelle en grande partie déconnectée du temps long, du «vrai» temps qui se présente sous le double aspect du temps de l'histoire et de la temporalité existentielle de nos vies respectives. Quant aux groupes eux-mêmes, qui déploient leur «dynamique», ils relèvent du paradigme de l'espace. Non seulement, bien sûr, le stage est résidentiel, en un lieu donné, mais encore, sans forcer les choses, on peut y voir la métaphore d'un îlot de spatialité, au sens des espaces culturels et linguistiques mis «en contact» (pour parler comme Weinreich) et, plus encore, au sens de la localisation dans un espace relationnel et situationnel plus restreint qu'il fait exister, pour un temps... En somme, on a affaire à une double localisation: une macrolocalisation dans l'espace de deux cultures et de deux langues, et puis une microlocalisation dans l'espace restreint d'un groupe binational. À quoi on pourra ajouter que les animateurschercheurs se trouvent «localisés» par immersion dans le groupe. Dans un tel contexte, la localisation reprend le sens large, pour ainsi dire déterminologisé et ré-idiomatisé, qui est traditionnellement le sien dans la langue. 
Il est clair que le recours à un interprète compétent, c'est-à-dire professionnel et donc nécessairement extérieur au groupe, par sa provenance et du fait de la fonction apparemment «neutre» qu'il est censé assurer, était incompatible avec cette recherche ayant pour objet d'examiner comment se produit la communication de groupe à la base ou, comme on dit, "sur le tas». C'est ce que le dispositif spécifique de l'interprétation aurait rendu impossible, par construction. Ne fût-ce que parce que, en raison de ce qu'on pourrait appeler un effet de "communication paradoxale» (au sens de l'école de Palo Alto), le formalisme d'une traduction systématique tend à rendre impossible les échanges qu'elle a pour fonction de promouvoir (Ladmiral 1982: 204). En effet, elle va se trouver occuper une place prépondérante, elle va induire des retards inhibant le vécu groupal, bloquer les possibilités de dialogue spontané, introduire des contresens et des malentendus, etc. Sans parler du fait qu'à l'inverse elle va tendre à alimenter le fantasme ou l'«illusion de la transparence traductive» (Ladmiral 1994: 230 et suivantes). Bien plus, cette illusion tend à déposséder les individus de la parole qui leur est propre et contribue à leur faire perdre la conscience des réalités qui concerne leur rapport au langage, avec ses contingences et ses pesanteurs, mais aussi ses limites, ses opacités, ses «trous»... Alors que, pour les «bilingues», le clivage des langues tendrait à mettre en évidence cet aspect des choses et à l'accentuer. On en vient à être prisonnier d'une illusion de la transparence langagière.

C'est donc au groupe lui-même qu'il reviendra de prendre en charge la traduction, c'est-à-dire en fait à ceux qui dans le groupe s'en sentent la capacité en général et en ont la volonté au moment venu. Ce service de communication devra donc être négocié (comme on "négocie» un virage au volant de sa voiture ou à skis) au coup par coup, en fonction du désir et des disponibilités de chacun, de la conjoncture dans le groupe, etc. Ladite traduction devient alors un phénomène de groupe: en plus de sa nature linguistique et interculturelle, elle se charge de surdéterminations psychosociologiques (le trait d'union est voulu).

Mais, à vrai dire, peut-on encore parler vraiment de traduction? Plus précisément, cette forme de «traduction orale» mérite-t-elle d'être qualifiée d'interprétation? Ou, comme préfèrent encore dire certains, d'interprétariat? À l'évidence: non! en raison des aléas qu'y introduisent la psychologie des individus concernés et la dynamique du groupe lui-même. Il en résulte que ce mode de communication interlinguistique sera sélectif et lacunaire, subjectif et orienté. Toujours est-il que c'est quand même le mot de «traduction» (et, en allemand, Übersetzung) qui est spontanément utilisé par les participants des différents stages. Il y a là une sorte de courant idiomatique sous-jacent et spontané, plus fort que notre volonté rationnelle d'endiguement terminologique du terme dans le canal de définitions précises, au sein du milieu spécialisé qui est le nôtre. C'est pourquoi j'ai repris ce mot à mon compte, dans ce contexte groupal - en dépit de tout ce que j’ai pu dire et écrire en tant que traductologue (et traducteur).

D'aucuns ne seront pas infondés à penser qu'il y a là un glissement de sens abusif. Mais toute l'histoire des langues n'est-elle pas celle des incessantes dérives sémantiques qui s'y produisent, comme nous le rappellent au demeurant les délices et les prestiges de l'étymologie? Au vrai, on n'a là en fait qu'une extension de sens qui fait du concept de traduction un générique; et cette logique de la synecdoque est inhérente au fonctionnement de tout lexique et de tout langage. Dans cet esprit, il ne me semble pas nécessaire de chercher un générique particulier (si je puis dire) pour y subsumer 
à la fois la traduction (stricto sensu) et l'interprétation, par exemple, comme le font certains auteurs de langue allemande qui entendent acclimater l'anglicisme Translation (au sein de leur Translationswissenschaft) pour en faire un néologisme terminologique investi de cette signification générique, en allemand. Dans cet esprit, j'ai d'emblée fait une place à l'emploi un peu erratique qui nous occupe ici dans le cadre du survol récapitulatif de la polysémie du concept de traduction que j’ai esquissé au début. Et puis il existe quand même une réelle continuité sémantique avec le ou les sens propres dont on peut exciper et l'usage qui est fait du mot dans la communication de groupe. Il reste que j'aurais pu - et sans doute aurais-je dû - mettre ce terme "traduction" entre guillemets dans l'intitulé de la présente étude (et si je ne l'ai pas fait, c'est qu'il n'est jamais bon de surcharger un titre de signes diacritiques).

\subsection{Traduction et médiations}

Cela dit, si le signifiant "traduction" doit pouvoir continuer à s'employer dans le cadre tout à fait particulier de la dynamique des groupes bilingues, le signifié qui lui est spécifiquement attaché dans ce contexte pourra renvoyer pour nous à des appellations différentes. En réalité, il s'agit proprement d'une médiation au sens plein du terme (Ladmiral et Lipiansky 1989: 46).

Mais il est vrai que ce concept est sans doute trop abstrait et polysémique, et que son sens n'est pas assez transparent pour qu'il puisse en l'espèce être substitué à celui de traduction. Encore que l'examen qu'il y a lieu d'en faire conduira à mettre en évidence une certaine continuité conceptuelle entre ce qu'est proprement la traduction et ce qu'on persiste à appeler «la traduction» dans le cadre de la dynamique des groupes bilingues.

De fait, la médiation traductive est multiple et se situe à plusieurs niveaux. D'une façon générale et à un niveau immédiat, la traduction est une médiation interlinguistique (1) qui fait passer un message d'une langue source ou langue originale (Lo) à une langue cible ou langue de la traduction (Lt). En outre, il n'est plus guère nécessaire d'insister sur le fait que cela va nécessairement de pair avec une médiation interculturelle (2), tant il est vrai que le passage d'une langue (Lo) à une autre (Lt) englobe, parallèlement et simultanément, le travail d'une médiation qui opère d'une langueculture (LCo) à une autre (LCt). Voilà un point qui a été abondamment thématisé ces dernières années dans le discours traductologique, dans le cadre du fameux «tournant culturel» de la traduction.

Plus intéressant peut-être est de rappeler que la traduction est en même temps ce que j'appellerai une médiation langagière (3). En insistant sur le fait qu'il ne s'agit pas tant de traduire le texte lui-même, dans la matérialité de ses signifiants, que le vouloir-dire dont il est porteur, Danica Seleskovitch et Marianne Lederer avaient affranchi la traductologie de la tyrannie méthodologique de ce que je me plais à appeler la linguistique de naguère (Seleskovitch et Lederer 1984). Ce n'est pas le lieu ici de faire un sort à ce concept qui a fait l'objet de diverses critiques, mais qui a le mérite de pointer dans la bonne direction. Il n'est pas jusqu'à la part de flou qu'il comporte qui n'ait une valeur réellement heuristique, comme j'ai cru bon de le dire lors d'un colloque de l'ÉSIT (Ladmiral 2002a) et ailleurs. Dans cette formule, il y a le télescopage de deux significations lexicoconceptuelles finalement assez différentes: vouloir dire, cela peut... "vouloir dire» tout simplement ce que signifie un énoncé 
(what it means); à moins qu'on ne prenne à la lettre l'expression et qu'elle «veuille dire» ce qu'un sujet parlant veut dire, ce qu'il a la volonté d'exprimer (what he wants to say), ce qu'il a en tête (intentio dicendi). Dans le premier cas, on en est resté au plan de la pure et simple communication linguistique; et c'est à cette première signification qu'on pense en matière de traduction. Mais la seconde fait déjà dériver la médiation langagière vers ce que le positivisme de la linguistique de naguère dénonçait sous le nom de «mentalisme», vers cette zone floue où le linguistique confine au psychologique. Pour reprendre une formule que j'affectionne: le traducteur n'a pas à traduire ce qui est écrit, mais ce qu'il pense qu'a pu penser celui qui a écrit ce qu'il a écrit quand il l'a écrit (Ladmiral et Lipiansky 1989: 53). On ne peut pas faire plus simple!

Cela vaut pour la traduction en général. Mais il apparaît que le dispositif psychosociologique de la dynamique des groupes bilingues (et interculturels) tend à faire émerger dans le prolongement de ladite médiation langagière plusieurs autres modalités de médiations d'ordre effectivement psychologique. Ainsi, à un niveau élémentaire, si l'on va plus loin dans le sens du vouloir dire (sans trait d'union) au sens d'une réelle volonté de s'exprimer, cela nous renvoie à ce qu'on pourrait appeler une médiation psycholinguistique (4): cela attire notre attention notamment sur les décalages qui ont pu venir s'insinuer entre ce qu'un sujet a pu dire et ce qu'il avait voulu dire. D’où ces nombreux « ratés de la communication» que constituent les lapsus, les simples bafouillages et autres malentendus... Il y a là des effets dyscommunicationnels que la dynamique du groupe va contribuer à provoquer plus abondamment que d'ordinaire en raison de la pression qu'elle peut induire chez les individus, des tensions conflictuelles qui pourront se manifester entre les participants, ou même de la simple difficulté à s'exprimer en public dans un tel contexte, etc. En forçant à peine les choses, on peut dire qu'ici le vouloir-dire représente une forme de localisation entre deux synecdoques, au sens où il s'agit en quelque sorte de "localiser» un contenu mental, dont les limites sont malaisément assignables, dans les limites d'un énoncé fini (pars pro toto), dont la signification fait signe vers les vastes horizons de toute une expérience du monde partagée (totum pro parte). On rejoint la réflexion de Marianne Lederer sur les synecdoques différentielles par lesquelles doit passer la parole (ou le discours) pour aller d'une langue (Lo) à une autre (Lt). Ces réflexions d'ordre général intéressent le traductologue (Seleskovitch et Lederer 1984) autant que le philosophe.

Plus spécifiquement, on ne s'étonnera pas que, dans le cadre de la dynamique des groupes bilingues, la "traduction» donne lieu aux diverses modalités d'une médiation psycho-sociologique. D’une façon générale, Henri Meschonnic a marqué avec force qu'il ne s'agit pas de traduire ce que le texte dit, mais ce que le texte fait (Meschonnic 1999: 22, 55, 124, 139, etc.). Dans la perspective qui était la sienne, cela concernait les grands textes, bibliques et littéraires. Mais c'est aussi une formule parfaitement adéquate aux différentes formes que peut prendre la traduction professionnelle ou spécialisée, comme l'ont noté Christian Balliu ou Nicolas Froeliger. Quant à moi, dans le contexte de la communication de groupe dont je traite ici, j'irai plus loin. Le vouloir-dire prendra ici le sens proprement littéral d'une médiation psychorelationnelle (5): il ne s'agit pas tant de traduire ce qu'un participant a dit - ce qu'il a "voulu dire» - que de réexprimer dans l'autre langue ce qu'il veut! Ce qu'il veut tout court, ce qu'il désire. On s'est éloigné sensiblement de ce qu'est une traduction (stricto sensu). Celui qui s'est assigné la tâche de médiation de communication 
va réinsérer dans sa «traduction» du contenu verbal (ou, comme on dit, "parolique») de l'intervention d'un participant au sein du groupe à la fois ce qu'il sait de la personnalité et des états d'âme de ce dernier; et cela implique la relation qu'il entretient avec lui, ainsi que tout un non-dit déterminé par les données situationnelles du moment propre à la dynamique du groupe et à son histoire préalable. En somme, la dite traduction aura procédé à une relocalisation psychorelationnelle et groupale des contenus d'énonciation.

Mais, dans la logique de mon propos ici, l'importance la plus manifeste revient à la traduction envisagée dans la perspective d'une médiation psychosociologique (6) - que j'écris maintenant sans trait d'union, car ce n'est plus un adjectif composé (pour ainsi dire), au signifié holiste, mais l'adjectivation de ce syntagme nominal qui est l'appellation consacrée d'une des disciplines au sein de la galaxie de ce qu'il est maintenant convenu d'appeler les «sciences psychologiques", à savoir la psychologie sociale (ou psychosociologie), dont la dynamique de groupe est elle-même une sous-discipline. La question a déjà été abordée plus haut, quand j'ai expliqué pourquoi il convenait d'écarter la solution technique de l'interprète pour assurer la communication dans le cadre de la dynamique des groupes bilingues, d'une part, et à propos de la dimension groupale inhérente à la médiation psychorelationnelle que je viens d'évoquer, d'autre part. J’y reviendrai de façon un peu plus concrète et détaillée à la fin de la présente étude; mais j'entends auparavant faire le point, très succinctement, sur le plan épistémologique.

\subsection{Remarques épistémologiques}

Il y a lieu en effet de s'interroger sur le statut «métathéorique» de la recherche à laquelle font écho les analyses proposées ici. Dans cet esprit, je m’en tiendrai à trois remarques, qui resteront programmatiques.

Premièrement: avant toute chose, j'entends marquer très nettement la spécificité épistémologique des sciences humaines, par opposition à l'attitude positiviste, qui voudrait les identifier à toute force aux sciences exactes, partant du principe qu'il n'est de science que ces dernières. Et encore je ne reprendrai pas ici la critique $d u$ positivisme que je poursuis depuis plusieurs années dans mes divers travaux, où je me suis attaché à en dégager les développements philosophiques et les implications politiques prolongeant la réflexion épistémologique. Sans entrer dans les détails, il convient seulement de rappeler que dans nos disciplines - qu'on semble maintenant préférer appeler les sciences sociales et humaines de façon, à vrai dire, un peu redondante -, on est très généralement assez loin de la méthode expérimentale et de la formalisation logico-mathématique, hormis quelques spécialités à la marge (comme la psychologie expérimentale). La formalisation du discours y est essentiellement un mode de présentation et ne saurait prétendre à une rigueur proprement scientifique. Et pourtant, il y a production de connaissances, mais sur un mode épistémologique qui leur est propre. Cela dit, il n'est que trop clair que ce n'est pas le lieu ici de développer (ni même d'esquisser) une épistémologie des sciences humaines.

Deuxièmement: s'agissant de «mon» programme de recherche sur la dynamique des groupes bilingues et binationaux, il était censé relever de la recherche appliquée, conjointement avec tout un ensemble d'autres programmes. Il m'apparaît qu'il y a lieu de s'interroger sur cette étiquette conceptuelle. Ầ première vue, il semblerait qu'il 
y ait là une contradiction dans les termes. L'idée d'«application» désigne en l'occurrence le fait qu'il est assigné des objectifs plus ou moins déterminés préalablement au déroulement de la recherche elle-même. Or le propre de la recherche, c'est sa vocation heuristique, c'est-à-dire qu'elle a pour fonction de découvrir quelque chose de nouveau, qui n'était pas prévu ni même prévisible. Faute de quoi, ce n'est pas de la recherche, mais de la pédagogie, de l'apologétique, de l'idéologie... En fait, comme c'est souvent le cas, une contradiction logico-conceptuelle va se résoudre au niveau de la langue en effets particuliers de sémantisation minimaliste. Dans le domaine technologique, la recherche est censée déboucher effectivement sur des "applications", utilisables à plus ou moins court terme. En matière de traduction, on parle volontiers de recherche appliquée pour signifier que la théorie traductologique a pour fonction principale sinon exclusive de fournir une aide à la pratique traduisante. Mais la formule peut correspondre aussi à des surdéterminations institutionnelles et je pense que c'est en partie la raison pour laquelle Ewald Brass, le responsable commanditaire des recherches menées sous l'égide de l'OFAJ, y a eu recours : le projet de (ne) faire (que) de la «recherche appliquée» étant de nature à rassurer les bailleurs de fonds des autorités de tutelle, ou du moins à ne pas les affoler... De fait, ces différents angles de vue tendent à se rejoindre, en particulier pour la traduction et la dynamique des groupes bilingues, dans le cadre de laquelle se localise la «traduction» dont je traite ici.

Troisièmement: plus précisément, pour qualifier l'approche méthodologique mise en ouvre dans le programme de recherche dont il est question, la formule la plus adéquate est le concept de recherche-action (Barbier 1996). Comme on sait, c'est une méthodologie qui a été développée en psychologie sociale et en sciences de l'éducation notamment, et qui trouvait tout naturellement à s'appliquer (doublement) dans le cadre du programme de recherche évoqué ici. Dans cette perspective, je distingue au sein des sciences humaines un sous-groupe à part constitué par les «sciences de l'action» ou (comme je préfère les appeler) les praxéologies: dans cette rubrique, on pourra ranger une grande part de la psychologie sociale et des sciences de l'éducation, donc, mais aussi l'économie d'entreprise et les dites «sciences de la gestion» - et j'y ajouterai la traductologie. Or on retrouve là encore le paradoxe d'une contradiction apparente. En un mot: ce paradoxe épistémologique consiste en ceci que le chercheur qui s'attache à faire la théorie prenant pour objet un pan de la réalité intervient au sein même de cette réalité qu'il prétend décrire. C'est très précisément le cas pour le champ d'études qui nous occupe, à un double titre: tant pour la dynamique des groupes bilingues, où l'animateur-chercheur est immergé dans le groupe, que pour la «traduction» à laquelle elle donne lieu (voir l'anthropologie participative).

À quoi on sera fondé à objecter qu'il y a là un mélange entre le sujet connaissant et l'objet connu (à connaître), ce qui n'est pas épistémologiquement correct. La réponse à l'objection est à double détente en quelque sorte. D’abord, cela correspond à la réalité! C'est un fait incontournable dont on ne peut pas ne pas partir - quitte, bien sûr, à recourir à certaines médiations méthodologiques permettant d'objectiver les phénomènes étudiés. Mais les procédures d'objectivation expérimentales qui pourront être utilisées déboucheront sur une artificialisation génératrice d'artefacts qui vont induire des perturbations au sein de l'objet étudié; et elles constituent, à leur tour, une intervention de nature subjective. Mais ce n'est pas le lieu de développer ici la controverse épistémologique opposant expérimentalistes et cliniciens, qui 
nous éloignerait de notre propos et dont j'ai amplement traité ailleurs (Ladmiral 2002b et 2008).

Pour être plus précis, je dois reconnaître que c'est essentiellement dans la perspective qui est la mienne que la traductologie relève vraiment de la recherche-action, c'est-à-dire qu'il s'agit de ce que je me suis attaché à définir comme la traductologie productive, qui s'efforce d'apporter une aide au traducteur aujourd'hui. En réalité, la plupart des recherches en la matière restent encore des études sur la traduction (Translation Studies) qui se réclament de méthodologies objectivantes, qu'il s'agisse de ce qui à mon sens constitue soit la traductologie descriptive, soit la traductologie inductive ou scientifique. La première s'en tient en effet à la description purement linguistique (et stylistique) objectivant l'après-coup de la démarche de traduire et procède à des études contrastivistes comparant un texte (To) et sa traduction ( $\mathrm{Tt}$ ) et éventuellement ses traductions (Tt, Tt', Tt”, Tt"'...). Pour moi, c'est la traductologie d'hier; et il convient d'aller dans le sens de ce que j'ai thématisé dans les termes d'une «déconstruction du linguistique» en traductologie. La seconde s'attache à mettre sur pied une étude proprement scientifique du phénomène de la traduction et des opérations dont elle procède, sur la base d'une méthodologie objectiviste faisant appel aux sciences cognitives. Sans parler des critiques de fond qu'il y aurait lieu d'en faire (et auxquelles je consacrerai une prochaine étude), il convient de souligner d'entrée de jeu qu'on n'en a encore que des linéaments dispersés et j'y vois quant à moi la traductologie de demain ou même plutôt d'après-demain.

J'ai dû me limiter ici à un survol de la question, prenant la forme de ce qui pourra sembler à certains n'être que des formules à l'emporte-pièce: je ne pouvais pas en effet reprendre par le menu les analyses touchant l'épistémologie de la traduction dont j'ai traité dans plusieurs de mes travaux (dont je ne donnerai qu'une référence: Ladmiral 2010).

\section{Perspectives pratiques}

Dans le contexte de la présente étude, la communication interculturelle se concrétise comme une forme tout à fait particulière de «traduction» localisée dans la dynamique psychosociologique du groupe bilingue et dans la parole de ses participants. Corollairement, elle fait donc l'objet d'une localisation spécifique dans l'oralité de la communication de groupe qui est ainsi mise en œuvre.

La médiation de cette communication orale que constitue le dispositif groupal de «la traduction» devient un enjeu relationnel, où interfèrent les rapports de pouvoir, de séduction, les clivages idéologiques, socioprofessionnels ou nationaux, les enjeux d'argent, les rapports intellectuels et corporels, les tensions conflictuelles entre individus et entre sous-groupes plus ou moins "communautarisés», etc. Le caractère «volatil» de l'oralité induit en effet une fluidité extrêmement changeante dans les relations entre individus et dans la redistribution plus ou moins constante des sousgroupes. Le caractère imprévisible d'une oralité spontanée, réagissant à des situations instables et inopinées, dans l'instant, ouvre la voie à des paroles inconsidérées, mais aussi à toutes sortes de dérapages - et notamment à l'émergence des conflits, comme aussi à la désinhibition des comportements sexuels.

On peut dire que, dans le contexte groupal, l'oralité est un accélérateur de la communication, qui pourra exacerber les enjeux du désir, de l'agressivité, de la relation 
dominant/dominé, etc. Pour les individus, il en résultera des effets de déstabilisation personnelle, de jouissance hystérique, de névrose d'abandon, ainsi que des demandes fusionnelles, des fantasmes paranoïdes et autres, etc. Il pourra même se faire que certains utilisent le travail de groupe pour leur propre psychothérapie personnelle, tant il est vrai qu'en fin compte on a quand même affaire à des "groupes de thérapie» (T-group). D'autres, voire les mêmes, chercheront à se fuir, c'est-à-dire à se décentrer de leur propre moi, en se précipitant vers une forme d'escapisme idéologique consistant à militer pour telle ou telle des innombrables «causes» qu'il y a sur le marché de l'idéologie régnante: antiracisme, féminisme, tiers-mondisme, régionalismes, combat pour diverses "minorités» (homosexuelle, noire, "beure»...), messianismes religieux, militantismes proprement politiques, diverses versions oblatives du déni de soi-même, etc. Sans parler des stratégies d'alliance ou de rupture, auxquelles l'oralité mise en scène dans le groupe (et devant le groupe) confère un caractère théâtral (et parfois cathartique). Dès lors que la «traduction» (comme tenaient à dire les participants) est prise en charge au sein du groupe sur la base du volontariat, ainsi qu'il a été indiqué, elle devient l'affaire du groupe, une affaire de groupe - où va s'engouffrer l'ensemble des pesanteurs et des appétences qui viennent d'être évoquées, lesquelles vont surdéterminer le travail de médiation interlinguistique. Elle est devenue un enjeu groupal de pouvoir, de séduction, de marchandages divers... D'une façon générale, l'oralité omniprésente débouche sur une inflation du mode «parolique» de relation et d'existence.

En matière de dynamique de groupe - un peu comme en histoire ou en psychanalyse -, il est finalement très difficile de prévoir ce qui va se passer, mais il est possible de repérer et d'analyser (au moins rétrospectivement) certaines séquences dans le déroulement du vécu du groupe. C’est ce que j'appelle des scénarios de la communication interculturelle et interlinguistique de groupe, lesquels se révèlent être très variés. Cela se conçoit compte tenu de l'extrême complexité des phénomènes mis en jeu. Outre la dynamique psychosociologique du groupe lui-même, avec toute l'incertitude de ses fluctuations, il y a la complexité propre à chaque individu et aux comportements qu'il se trouve adopter dans la situation. Dans la conjoncture de la dynamique des groupes bilingues, il intervient de surcroît la variable du clivage des langues et la dimension interculturelle, cette dernière jouant à plusieurs niveaux. D'abord, il est clair qu'il y a un capital culturel qui s'est pour ainsi dire sédimenté dans la langue elle-même, dans son lexique, dans ses tournures et sa phraséologie, dans l'implicite de la discursivité qu'elle peut déployer, etc. Parler une langue, c'est adopter un mode de pensée et faire sienne une culture. En outre, les individus que nous sommes tous ne peuvent pas plus échapper totalement à leur milieu social d'origine ou à leur appartenance générationnelle qu'aux déterminations de la culture qu'ils ont intériorisée. Et puis, plus généralement, il existe tout un non-dit flottant de références culturelles diffuses, mais omniprésentes dans le collectif d'un groupe (en situation) et d'une société (sur un temps plus long).

Je ne vais pas reprendre ici les «scénarios» que j’ai déjà exposés en d'autres lieux (Ladmiral et Lipiansky 1989). Je veux seulement conclure sur quelques esquisses indicatives.

Quand, par exemple, il nous est demandé de traduire nous-mêmes dans l'autre langue (Lt) les propos que nous venons de tenir dans l'une des deux langues (Lo), quand nous nous traduisons donc nous-mêmes, il n'est pas rare que nous nous trou- 
vions entraînés dans ce que j'appellerai une dynamique d'auto-incrémentalisation interne de la traduction. En effet, compte tenu des décalages interculturels que met en évidence le clivage des langues et auxquels il incombe à un bon traducteur d'apporter des compensations explicitatives, et compte tenu par ailleurs de la plus-value sémantique que tend à induire le travail psycho-existentiel et psychorelationnel de la verbalisation, on est porté par la logique propre de l'autotraduction à clarifier et à développer dans sa traduction (Tt) des points qu'on avait laissés dans l'ombre (ou dont l'importance avait échappé) dans ce qu'avait été l'intervention originale au départ (To). Il en résulte un dépassement incrémentiel dans ladite traduction. Mais il convient dès lors de retraduire dans la langue de départ (Lo) ce qu'on y a rajouté. Par exemple: je dis quelque chose en français (To); et j'en dis plus dans la traduction que j'en fais en allemand ( $\mathrm{Tt}$ ); il n'y a donc pas de raison que je ne retraduise pas en français (To') ce que j'ai rajouté en allemand; mais pourquoi faudrait-il que je ne re-re-traduisisse pas en allemand (Tt') un autre complément au second degré que j'aurai pu encore rajouter en français... De proche en proche, on en viendrait à une inflation indéfinie de la traduction dans le vase clos d'un autocentrement sur une ipséité interne:

$$
\mathrm{To} \rightarrow \mathrm{Tt} \mathrm{To} \rightarrow \mathrm{To} \text { To } \rightarrow \mathrm{Tt}^{\prime} \rightarrow \mathrm{To}^{\prime \prime} \rightarrow \mathrm{Tt}^{\prime \prime} \rightarrow \mathrm{To}{ }^{\prime \prime} \rightarrow \mathrm{Tt}^{\prime \prime}
$$

sic in infinitum! Dans les faits, il faut que ça s'arrête, bien sûr. Cela dit, il reste que cette dynamique incrémentielle de l'autotraduction n'est pas qu'un débordement narcissique du traducteur: cela correspond à l'attente des unilingues, qui souvent veulent «ne pas en perdre une miette» (au moins dans certains cas), sous peine de se sentir exclus de la communication, pour ainsi dire «excommuniés»...

Plus surprenant peut-être est un autre scénario qui peut survenir, encore une fois en cas d'autotraduction. Quand on est amené à traduire soi-même son intervention, il arrive plus souvent qu'on le croirait qu'on réexprime effectivement ce qu'on vient de dire avec d'autres mots, mais dans la même langue! Et ce, sans s'en rendre compte, jusqu'à ce qu'un éclat de rire dans le groupe (ou une protestation) nous en fasse prendre conscience. C'est l'occasion de mesurer que la médiation qu'opère cette forme de «traduction» (à laquelle j'avais cessé de mettre des guillemets par commodité) nous rappelle qu'au sens propre la traduction et l'interprétation (dont la dite «traduction» est ici est une illustration particulière) ne posent pas seulement ni même principalement un problème d'ordre purement linguistique, mais qu'elles relèvent fondamentalement de la psychologie. Le travail de l'interprète procède en effet d'abord d'un effort de mémorisation immédiate des contenus énoncés en langue source et d'un travail cognitif de conceptualisation préalablement à la réexpression en langue cible, étant entendu que «l'intendance» des langues suivra! dirai-je, pour paraphraser une formule de Charles de Gaulle (Ladmiral 2005). Sauf qu'en l'occurrence il peut arriver parfois qu'on se trompe de langue... Et paradoxalement, ce genre de cafouillage valide le modèle.

Il est bien d'autres cas de figure, où tout un ensemble de déterminations pourront interférer avec «la traduction»: les enjeux psychorelationnels, les stratégies individuelles, la façon mouvante dont tout cela se «localise» dans l'espace groupal à un moment donné, sans oublier des éléments de transversalité divers qui peuvent provenir de l'écosystème global où s'insère le groupe, etc. Un exemple rapide, où ce n'est pas seulement le fonctionnement psychocognitif de la traduction qui surdétermine 
la médiation linguistique, mais des données relevant d'un fonctionnement psychologique plus global. Je te traduis parce que je t'aime: parce que je veux te plaire, te séduire peut-être, ou parce que je te sens fragile ou en difficulté, et que je veux te marquer ma sympathie, t'aider, te sortir de l'isolement... Ou, au contraire, je te traduis parce que je ne t'aime pas: parce que je veux bien faire ressortir la sottise et l'arrogance de tes propos aux yeux de tous! Dans les deux cas, la traduction sera tendancieuse et va accompagner la relation psychorelationnelle qui la sous-tend, en forçant le trait dans un sens ou dans l'autre. Là, l'enjeu est relationnel, c'est-à-dire essentiellement lié aux rapports que les individus entretiennent entre eux, mais aussi au contexte situationnel et à la configuration momentanée de la dynamique du groupe.

Je pourrais multiplier les exemples à l'envi, au risque de lasser le lecteur (que je renvoie pour plus de détails concrets aux études déjà publiées). En effet, le champ d'études de la clinique - qu'elle soit médicale, psycho-pathologique, psychanalytique, psychogénétique ou, donc, psycho-sociale - est par définition sans limites. Un peu comme la demande thérapeutique, à laquelle il lui arrive de devoir faire face, est une demande infinie... ${ }^{2}$

\section{NOTES}

1. À quoi nous a rappelé Philippe Rothstein lors de la Journée de la traductologie de plein champ, le 12 septembre 2009: <http://www.eila.univ-paris-diderot.fr/recherche/conf/ciel/traductologieplein-champ/index $>$. Consultée le 12 septembre 2010.

2. On aura noté que, conformément à un usage de plus en plus répandu dans les publications en sciences humaines, et qu'on pourra trouver agaçant, j'ai été amené à citer plusieurs de mes propres travaux. La présente étude s'inscrit en effet dans le cadre d'un programme de recherche et dans la logique d'une réflexion d'ensemble, dont c'était l'occasion de faire apparaître la cohérence; et il m’a semblé utile d'en marquer certains points de contact. C'était aussi une façon d'alléger cette même étude qui, avec le recul, menaçait de prendre une ampleur excessive. Et puis, je suis quant à moi reconnaissant aux auteurs que je lis quand ils me fournissent des indications de cette nature, qui me permettent d'approfondir tel ou tel point.

\section{RÉFÉRENCES}

BArbier, René (1996): La Recherche-Action. Paris: Anthropos.

Culioli, Antoine (1990): Pour une linguistique de l'énonciation - Opérations et représentations. Vol. 1. Gap: Ophrys.

Culıoli, Antoine (1999a): Pour une linguistique de l'énonciation - Formalisation et opérations de repérage. Vol. 2. Gap: Ophrys.

Culioli, Antoine (1999b): Pour une linguistique de l'énonciation - Domaine notionnel. Vol. 3. Gap: Ophrys.

DeLisLe, Jean (1980): L'Analyse de discours comme méthode de traduction. Initiation à la traduction française de textes pragmatiques anglais. Ottawa: Éditions de l'Université d'Ottawa.

Froeliger, Nicolas (2009) : À quoi bon enseigner la traduction technique? In: Colette LAPLACE, Marianne Lederer et Daniel Gile, dir. Profession traducteur. Collection Cahiers Champollion. Paris-Caen: Minard-Lettres modernes, 199-210.

Ladmiral, Jean-René (1982): Traduction et psychosociologie. Meta. 27(2):196-206.

Ladmiral, Jean-René (1994): Traduire: théorèmes pour la traduction. Collection Tel. Vol. 246. Paris: Gallimard.

LADMiral, Jean-René (1995): Traduire, c'est-à-dire... - Phénoménologie d'un concept pluriel. Meta. 40(3):409-420.

Ladmiral, Jean-René (1997): Le prisme interculturel de la traduction. Palimpsestes. 11:13-28. 
LAdmiral, Jean-René (2002a): La traduction, un concept aporétique? In: Fortunato IsRael, dir. Identité, altérité, équivalence? La traduction comme relation. Collection Cahiers Champollion. Paris-Caen: Minard-Lettres modernes, 117-144.

LADMIRAL, Jean-René (2002b) : La traductologie au xxi siècle: de la linguistique à la psychologie. In: Traduire au XXI siècle: Tendances et perspectives, 336-346.

Ladmiral, Jean-René (2004): La traduction entre en philosophie. In: Antonio Lavieri, dir. La Traduzione fra filosofia e letteratura/La Traduction entre philosophie et littérature. Turin: L'Harmattan Italia, 24-65.

Ladmiral, Jean-René (2005): Le «salto mortale» de la déverbalisation. Meta. 50(2):473-487.

Ladmiral, Jean-René (2006): L'empire des sens. In: Marianne Lederer, dir. Le sens en traduction. Collection Cahiers Champollion. Paris-Caen: Minard-Lettres modernes, 109-125.

Ladmiral, Jean-René (2008): Pour un tournant œcuménique en théorie de la traduction. In: Christian BALliu, dir. Traduire: un métier d'avenir. Vol. 1. Bruxelles: Les Éditions du Hazard, 11-32.

Ladmiral, Jean-René (2010): Sur le discours méta-traductif de la traductologie. Meta. 55(1):4-14.

Ladmiral, Jean-René et Lipiansky, Edmond Marc (1989): La Communication interculturelle. Paris: Armand Colin.

Lavault-Olleon, Élisabeth, dir. (2007): Traduction spécialisée: pratiques, théories, formations. Collection Travaux Interdisciplinaires et Plurilingues en Langues Étrangères Appliquées, dirigée par Klaus Morgenroth et Paul Vaiss. Vol. 10. Berne: Peter Lang.

Meschonnic, Henri (1999): Poétique du traduire. Lagrasse: Verdier.

Reiss, Katharina (2009) : Problématiques de la traduction. (Traduit par Catherine A. Bocquet) Bibliothèque de traductologie. Paris: Anthopos/Economica.

Seleskovitch, Danica et Lederer, Marianne (1984): Interpréter pour traduire. Paris: Didier. 